\title{
La videoconferencia como herramienta digital para el trabajo colaborativo en la educación superior
} Videoconferencing as a digital tool for collaborative work in higher education

\section{A videoconferência como ferramenta digital para trabalho colaborativo no ensino superior}

\author{
Salvador Barrera Rodríguez \\ Universidad de Guadalajara, México \\ sachavir@cucea.udg.mx \\ https://orcid.org/0000-0002-6870-5379
}

\section{Resumen}

A través de este trabajo quiero ahondar en la evolución de las herramientas de videoconferencia, sus características, el uso pedagógico, su etiqueta, ventajas y desventajas. En especial quiero destacar las herramientas de Microsoft Skype, zoom, Google Meet y Cisco Webex. Describir la experiencia de su uso a nivel licenciatura, para clases de tecnología del área económico-administrativa de una Universidad Pública del Estado de Jalisco. En sus características revisar la opción para grabación de la sesión, establecer un fondo virtual, la compartición de pantalla y el salón de charla, la programación de sesiones. Así como recomendar herramientas para la grabación de tutoriales y cambio de avatar y fondo. El uso sugerido fue de una hora de retroalimentación y dialogo de preguntas generadoras (teoría) y otra hora de ejercicios colaborativos en OneDrive y otros servicios de internet, donde los alumnos demuestren sus competencias después de haber visto videotutoriales propios y de YouTube. La recomendación de entrar con la videocámara prendida y el micrófono apagado. Las dificultades técnicas y como disminuirlas. Recomendaciones para evitar el zoombombing, Experiencia sobre la duración de la sesión y el espaciado en las mismas. 


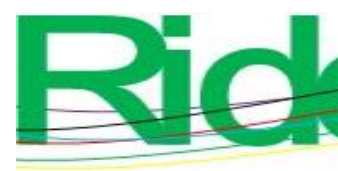

Revista Iberoamericana para la Investigación y el Desarrollo Educativo ISSN $2007-7467$

Palabras clave: Cisco Webex, educación pública, enseñanza asistida por ordenador, enseñanza superior, Google Meet, Microsoft Skype, tecnología de la información teleconferencia, video educativo, Zoom.

\section{Abstract}

Through this paper I want to delve into the evolution of videoconferencing tools, their characteristics, pedagogical use, their etiquette, advantages and disadvantages. I especially want to highlight the Microsoft Skype, zoom, Google Meet and Cisco Webex tools. Describe the experience of its use at the undergraduate level, for technology classes in the economicadministrative area of a Public University of the State of Jalisco. In its features, review the option to record the session, establish a virtual background, screen sharing and the chat room, and schedule sessions. As well as recommending tools for recording tutorials and changing the avatar and background. The suggested use was one hour of feedback and dialogue of generating questions (theory) and another hour of collaborative exercises in OneDrive and other internet services, where students demonstrate their skills after having seen their own video tutorials and YouTube. The recommendation to enter with the camcorder on and the microphone off. Technical difficulties and how to reduce them. Recommendations to avoid zoombombing, Experience on the duration of the session and the spacing in them.

Keywords: Cisco webex, public education, computer assisted instruction, higher education, Google Meet, Microsoft Skype, Information Technology, teleconferencing, educational video, Zoom.

\section{Resumo}

Com este trabalho, pretendo aprofundar a evolução das ferramentas de videoconferência, suas características, uso pedagógico, seu rótulo, vantagens e desvantagens. Quero destacar especialmente as ferramentas Microsoft Skype, Zoom, Google Meet e Cisco Webex. Descrever a experiência de sua utilização na graduação, para aulas de tecnologia na área econômico-administrativa de uma Universidade Pública do Estado de Jalisco. Em suas características, reveja a opção de gravar a sessão, estabelecer um fundo virtual, compartilhamento de tela e sala de chat e agendar sessões. Além de recomendar ferramentas para gravação de tutoriais e alteração de avatar e plano de fundo. $O$ uso sugerido foi uma hora de feedback e diálogo de geração de perguntas (teóricas) e outra hora de exercícios 


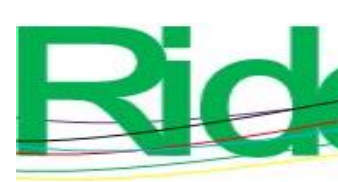

Revista Iberoamericana para la
Investigación y el Desarrollo Educativo
ISSN $2007-7467$

colaborativos no OneDrive e outros serviços de internet, onde os alunos demonstram suas habilidades após terem visto seus próprios vídeos tutoriais e YouTube. A recomendação para entrar com a câmera de vídeo ligada e o microfone desligado. Dificuldades técnicas e como reduzi-las. Recomendações para evitar zoombombing, Experiência na duração da sessão e no espaçamento entre elas.

Palavras-chave: Cisco Webex, educação pública, ensino assistido por computador, ensino superior, Google Meet, Microsoft Skype, teleconferência de tecnologia da informação, vídeo educacional, Zoom.

Fecha Recepción: Mayo 2020

Fecha Aceptación: Diciembre 2020

\section{Introducción}

Amaya Amaya (2010) diferencia entre teleconferencias, conferencias de datos y videoconferencias. La primera para conversaciones simultaneas de texto de programas de colaboración (groupware) o conferencias telefónicas múltiples, la segunda para edición de documentos en grupo (similar a lo que se puede hacer con Google Docs o Microsoft OneDrive) y en la tercera, en la cual, se pueden ver a sus interlocutores por pantallas de video son teleconferencias por video o videoconferencias.

En la investigación de la literatura he encontrado que inicialmente las videoconferencias se han usado para la educación a distancia, la educación en posgrado, sobre hardware y software especializado y costoso y luego se han diversificado a cada vez más ámbitos del conocimiento, niveles y tipo de enseñanzas. En la actualidad en estos tiempos de pandemia se consideraría una competencia básica del docente.

Para hablar de las posibilidades de la videoconferencia considero útil citar a Cabero Almenara \& Romero Tena (2010) donde menciona las ventajas, tipos y estrategias educativas: la mayor facilidad técnica, el costo que va bajando, desde usar hardware especializado para video, hasta el uso de software gratis para realizarlas, junto con el aumento de cursos que las usan. En tipos: hablan de conferencia punto a punto (propietaria) o multipunto. Grandes formatos con alta calidad, videoconferencia por la Red digital de servicios integrados y la videoconferencia por ordenador del tipo IP (internet). En cuanto a las estrategias educativas tenemos la exposición, la tutoría y retroalimentación.

Las Videoconferencias no son la única herramienta digital para la educación a distancia, tambien tenemos las plataformas de cursos en línea, pero las videoconferencias permiten una 

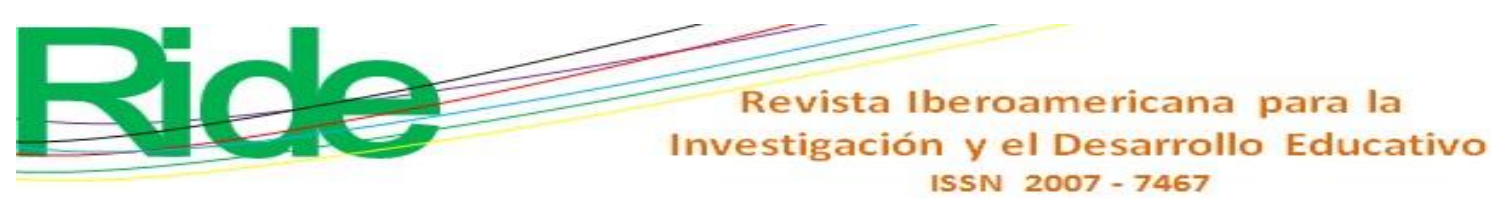

mejor retroalimentación y simulan mejor las clases presenciales además Castillo Holgado \& Fernandez Iglesias (2014) Nos habla de sus ventajas y usos:

- Una o múltiples sedes

- Compartir lo que ve la cámara. El ponente y su auditorio

- Compartir la pantalla

- Acceso remoto con algunas aplicaciones (tomar el control de la otra persona con su permiso)

- Compartir un pizarrón blanco para colaborar (característica que tiene Google Meet)

- Grabar las sesiones para compartir luego (y guardarlas en el momento como en zoom, Skype y Webex o que se comparta el enlace una vez procesadas como lo hace Google Meet)

- Se pueden distribuir ficheros (normalmente yo incluyo enlaces a documentos guardados en la nube para su visualización y/o descarga en la sala de conversación)

- Disponibilidad de un foro de conversación de texto o chat (muy útil si al alumno le falla el micrófono).

Sobre la utilización educativa de la videoconferencia mencionan

"Al contrario de lo que las personas creen, a la hora de utilizarla el profesor debe adoptar una serie de precauciones tanto en la preparación de las sesiones (tomar contacto con los equipos y programas que utilizará...) como en su desarrollo (cuidar el tiempo de intervención, utilizar diferentes estrategias didácticas...) y en los momentos de finalización con las actividades que desarrollará posteriormente.” (Barroso Osuna \& Cabrero Almenara, 2014) (p. 402).

En cuanto a los tipos distingue 3: las videoconferencias basados en la web, los que ocupan una extensión o plugin para funcionar, los basados en software de escritorio que ocupan una aplicación. (Y no mencionan aún las aplicaciones para dispositivos móviles).

Sobre las limitaciones de las videoconferencias el autor citado menciona también sus pros y contras, donde ahora mencionare los inconvenientes que indica:

1. El costo de los equipos y líneas usadas

2. La falta de experiencia de los profesores en su uso

3. Necesidad de un conocimiento mínimo de los alumnos y profesores en su uso (y un ingreso suficiente para que los pueda adquirir) 


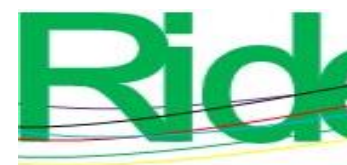

Revista Iberoamericana para la
Investigación y el Desarrollo Educativo
ISSN $2007-7467$

4. Preparación psicológica y didáctica para interaccionar tanto con los alumnos presenciales como con los remotos (los alumnos remotos se pueden sentir “olvidados" por lo cual cada cierto tiempo es conveniente interaccionar con ellos)

5. La calidad técnica de la imagen y sonido

6. Una buena preparación didáctica para conseguir la participación y el alumno no se aburra (normalmente se usa preguntas generadoras y archivos colaborativos para fomentar la participación, aquí es de gran ayuda la metodología del aula invertida).

El autor citado también explica que entre las aplicaciones educativas de la videoconferencia se encuentran: la educación a distancia consulta a expertos, proyectos multicentros, actividades personales y comunitarias. En las fases de preparación, desarrollo y extensión: habla de los preparativos del equipo, programas, materiales, planeación de la sesión. Durante el evento: tener variedad de actividades, moderar el tiempo, favorecer la participación y resumir al final. Concluir con las indicaciones de las tareas y revisión posterior.

El mismo Barroso Osuna \& Cabrero Almenara (2014) menciona los criterios para evaluar una conferencia: el funcionamiento de los equipos, las estrategias usadas, la coordinación del personal, la presentación del expositor, los materiales y el tiempo.

Lopez Curiel (2014) Enlista una serie de herramientas de Videoconferencia: FlashGoogle Meeting (que dejo de funcionar en 2018), Google Talk (que fue sustituido en 2013 por Hangouts, y en 2017 se dividió en Google Meet y Google Chat). La Aplicación zoom fue fundada en 2011. Cisco Webex esta desde 1995 y Microsoft Skype fue creado desde 2003. Hernández Adell (2016) Nos muestra su experiencia con la videoconferencia en la impartición de asignatura de Historia Económica Mundial y de España en una Universidad Virtual. Describe sus 3 materiales: Manual de la asignatura, E-learning, y presentaciones de la materia en PowerPoint. La duración de estas es de 10 sesiones de 2 horas. 2 sesiones por semana por 5 semanas. Una sesión por tema. Está programado desde el inicio el calendario de las videoconferencias y su objetivo es simular la experiencia presencial. Se graba la misma para su uso posterior. La usa para tutoría colectiva, presentaciones, seminario, ejercicios prácticos y su debate. Y la herramienta usada es Blackboard Collaborate, la cual tiene ventana de video, de chat, de participantes y una pizarra donde se presenta la pantalla de la presentación. Además, usa la aplicación socrative para dispositivos móviles y fomentar la interactividad mediante encuestas, cuestionarios, actividades. Y el propósito del autor de este articulo es documental su experiencia usando la videoconferencia a nivel licenciatura para clases de informática en este periodo de pandemia por el COVI-19. 


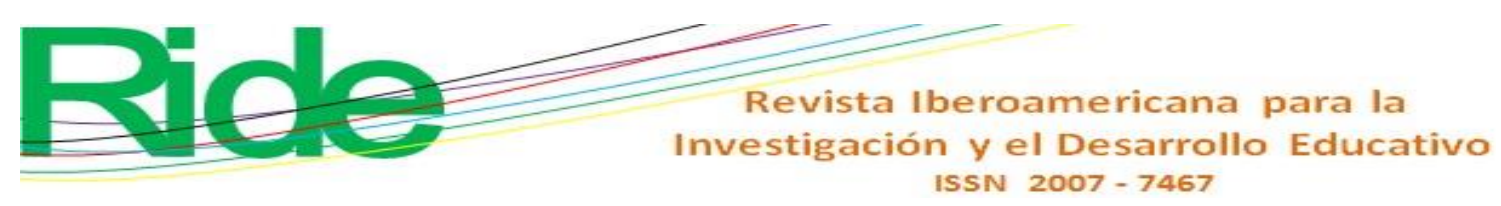

De acuerdo con su sitio web, Cisco Webex fue creado en 1995 por Subrah Iyar, Zhu Min en Milpitas, CA, E.U. permite hasta 100 participantes, video HD, sin límite de tiempo, pantalla compartida y sala personal en la versión gratuita.

Microsoft Skype, fue diseñado en 2003 por el danés Janus Friis y el sueco Niklas Zennström (también creadores de Kazaa) y desarrollada en su solución técnica por los estonios Priit Kasesalu, Ahti Heinla y Jaan Tallinn, ya que de hecho Skype nació en Tallin, Estonia. En 10 de mayo 2011, fue comprado por Microsoft.

En 2012 se anexo Windows Live Messenger. Y en marzo del 2014 termino el soporte a esas cuentas. En diciembre del 2013 Facebook ofrecía videollamadas a través de Skype. Este mismo año permitía videollamadas en grupo (antes solo dos personas). En verano del 2014, dejo de ser premium el uso de videollamadas en grupo y compartir el escritorio (hasta 10 personas). Antes del 5 de abril del 2019 el límite era 25 personas en videollamadas gratis, a partir de esa fecha permite hasta 50. En 2019, difuminar el fondo con Inteligencia Artificial. (También lo hace Zoom).

Zoom, fue fundado en 2012 por Eric Yuan, en San José, E.U. la versión gratuita tiene límites de hasta 100 personas y 40 minutos.

De acuerdo con García, (2020) (párr. 1).

"En la actualidad, las videollamadas de WhatsApp y las de Instagram son las que cuentan con el número de usuarios simultáneos más limitados, permitiendo solo cuatro. En comparación, FaceTime permite hasta 32 personas al mismo tiempo y Hangouts hasta 10 personas en su versión gratuita y 25 en la empresarial (Google Meet de G suite). Snapchat acepta hasta 15 personas, Facebook Messenger 50 personas (aunque solamente seis de ellas pueden retransmitir) y Duo permite realizar videollamadas de hasta 12 participantes."

Facebook Messenger, Desde enero del 2015 permite videoconferencias desde la versión de escritorio. Y desde el 28 de abril en dispositivos móviles como Celulares.

Evolución del Facebook Messenger: desde agosto del 2011 (como aplicación móvil)

- Llamadas: enero 2013 en Canadá y E.U.

- Videollamadas: 27 de abril 2015 (en algunos países, incluido México)

- Llamadas grupales hasta 50: 20 abril 2016

- Videollamadas grupales: hasta 50,19 de diciembre 2016.

- Reacciones animadas "efectos", capturas de pantallas y filtros. junio del 2017 


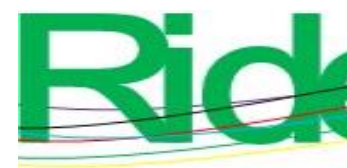

\section{Revista Iberoamericana para la Investigación y el Desarrollo Educativo ISSN $2007-7467$}

Primero fueron los sistemas dedicado de videoconferencias, luego los mediados por computadora, en la actualidad, los teléfonos inteligentes permiten comunicarse siempre y cuando se tengan datos o una conexión wifi. Enunciaremos a continuación la evolución de las conferencias en Whatsapp.

WhatsApp. Su característica de chat aparece en 2009. En agosto del 20013 los mensajes de voz. Fue comprado por Facebook en 2014:

- $\quad$ Llamadas de voz - desde febrero de 2015

- WhatsApp Web - desde agosto de 2015

- $\quad$ Grupos (hasta 256 miembros) - desde febrero de 2016

- $\quad$ Compartir documentos (.pdf, .docx, etc.) - desde marzo de 2016

- $\quad$ Videollamadas - desde octubre de 2016

- $\quad$ Videollamadas grupales (hasta 4) - desde julio de 2018

- $\quad$ Hasta 8. 20 abril 2020.

Según Brown (2020) Google Hangouts inicio en 2013 incluye la videoconferencia de Google plus Messenger y permito inicialmente hasta 10 usuarios convencionales, antes de la pandemia de COVID 19 permitía 25 usuarios empresariales y por el confinamiento hasta el 30 de septiembre del 2020, la versión gratuita toma prestadas características de Google Meet que permite hasta 250 personas y hacer transmisión de video en vivo hasta para 100,000 personas dentro de un dominio.

Google Duo, aplicación móvil y web de videoconferencia de alta calidad (HD), agosto de 2016. En abril del 2017 permite llamadas de voz. En diciembre del 2016 era el sustituto de Hangouts. Aunque luego salió Google Chat. Marzo 2018: mensajes de voz y video de hasta 30 segundos.

En mayo del 2019 se une a la competencia con hasta 8 usuarios simultáneos en 720p. FaceTime, WhatsApp, Skype and Facebook Messenger,

En G suite para la educación el programa Google Classroom permite el uso de Google Meet. El cual tiene versiones web y Android e iOS. Desde marzo 1917 surge como la versión empresarial de Google Hangouts. Presentado para hasta 30 usuarios y de acuerdo con su licencia: hasta 100 miembros por llamada para usuarios G Suite Basic hasta 150 para G Suite Business users, y hasta 250 con G Suite Enterprise users.

Microsoft Teams. La versión actual en 2017 sustituye a Skype empresarial (2015-2019), antes Microsoft Lync (2010-2013), antes Office Communicator 2007, antes Live 

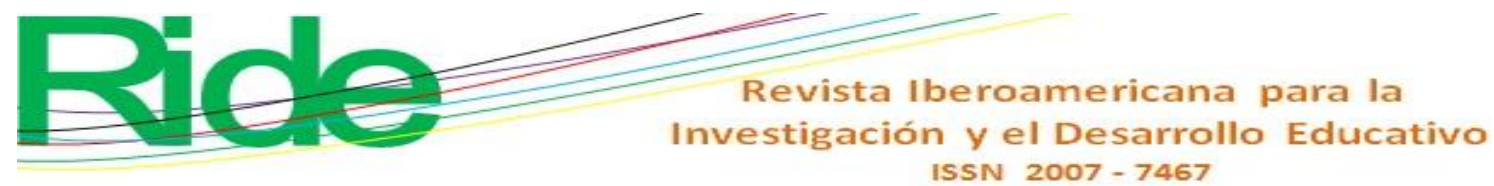

Comunication Server 2003 y 2005 que inicio como Exchange 2000 conferencing. Ha permitido pizarrón blanco, pantalla compartida, videoconferencias y encuestas.

Microsoft Teams es una plataforma unificada de comunicación y colaboración que combina una sala de conversación, reuniones de video, almacenamiento de archivos (incluyendo la colaboración en archivos) e integración de aplicaciones. El servicio se integra con el paquete de productividad de Office por suscripción y presenta extensiones que pueden integrarse con productos que no son de la compañía.

Microsoft presenta la versión educativa de Teams (2017) en junio 28 de ese año lanza la versión educativa de Microsoft Teams, que solo requiere una suscripción a Office 365.

\section{Objetivo}

Documentar la experiencia del uso de videoconferencia en la materia de tecnologías de la información, primer semestre, en una Universidad Pública del estado de Jalisco. Donde se inició $100 \%$ presencial y a partir del 21 de marzo del 2020, se cambió a $100 \%$ en línea, con motivo de la pandemia del COVID-19. La asignatura está apoyada en un curso diseñado en Moodle con recursos digitales, además de videotutoriales realizado con Loom. La Herramienta de videoconferencia utilizada Google Meet. Y la herramienta de colaboración fue la pantalla compartida, el chat y Microsoft Onedrive

Sobre los problemas a revisar serán: ¿la videoconferencia permite el traslado automático de una clase presencial a una virtual a Distancia?, ¿Qué dificultades presenta el uso de la videoconferencia en alumnos de licenciatura?, ¿en que difiere la educación a distancia con videoconferencia de las clases presenciales?

\section{Método}

Se emplea para este trabajo una investigación descriptiva, revisando los diferentes elementos que hacen posible la videoconferencia y los resultados obtenidos del uso de diferentes herramientas de colaboración como lo es videoconferencias por Zoom, Google Meet (y otras), Curso en línea por Moodle, colaboración por Microsoft Onedrive. Además de grabación de videotutoriales con la herramienta Loom. Bajo un paradigma constructivista. Se inicia el curso con un cuestionario diagnóstico contestado con un formulario de Microsoft forms donde se preguntó sobre su equipo, programas y conexión a internet y sus hábitos de estudio en línea. Las preguntas están disponibles en este enlace 


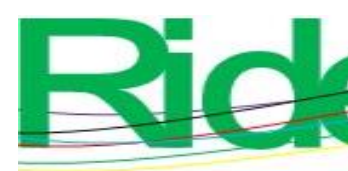

\section{Revista Iberoamericana para la Investigación y el Desarrollo Educativo ISSN $2007-7467$}

tener un fondo virtual, situación que con el programa Snap Camera y el mismo equipo, no es necesario.

Se continuo con la herramienta de Google Meet, donde genera una dirección diferente cada vez que se programa (para disminuir el riesgo de zoombombing o interrupción masiva y abusiva por parte de terceros ajenos a la reunión), si se crea una clase con Google Classroom, se puede disponer de una dirección fija, donde una vez programadas los horarios de sesiones no se tiene lugar a confusión. Se probó con un grupo con las sesiones programadas con diferentes enlaces y daba lugar a confusión del enlace correcto. Se tuvo una mala experiencia de compartir una dirección de la videoconferencia por la red social Twitter en vez del curso en línea de Moodle y entraron varios usuarios fuera de la institución y al aceptarlos el profesor, secuestraron “ en control de la pantalla, reproduciendo música en YouTube y video con sonidos indecentes, y como tratar de bloquearlos individualmente no era lo suficientemente rápido y salimos de la sesión y programamos otra, acordándolo desde los avisos de Moodle, en el curso en línea. Tiempo después, me di cuenta de que, usando el Navegador de Google Chrome, tenemos los controles del organizador para permitir si los alumnos pueden compartir la pantalla (lo cual en este curso por omisión no era necesario), incluso si podían escribir en el chat, lo cual si se dejaba abierto. Dado que se dejó como el equivalente al pizarrón y los avisos se anotaban en el chat.

Para la grabación hay varios asuntos, se deja programado que se va a grabar, y se manda un mensaje por correo cuando ya ha sido procesado y guardado, el nombre del archivo no se puede escoger de antemano y se tiene que renombrar si se maneja varias materias. Es muy importante que el profesor sea el último que salga de la reunión, de lo contrario se puede grabar los otros participantes hasta que ellos cuelguen, si no tienen imagen, ni voz se graba un recuadro negro por el tiempo que estén en la videoconferencia al final. Y por lo cual hizo necesaria su edición y recorte al publicar el enlace, para compartir la grabación para el tema puntual de las presentaciones. Otro detalle de la solución de G suite para la educación (que es la que permite el uso de Google Meet y de Google Drive, donde guardaron los videotutoriales), es que solo se puede ver con una cuenta de correo institucional de la misma, ofrece más privacidad, pero si los alumnos no entran con su cuenta institucional, pueden tener problemas de acceso.

Fondo virtual en Google Meet. Inicialmente se usó la herramienta de Snap Camera, para cambiar el fondo o incluso el avatar o presentador oficial. Se busco los más discretos, fondo borroso, blanco y negro y especialmente en el tema de las presentaciones en línea se fue más 


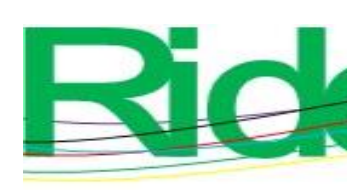

Revista Iberoamericana para la
Investigación y el Desarrollo Educativo
ISSN $2007-7467$

creativo, con otros que cambiaban incluso el avatar por una piña o un pretzel. Extrañamente no hubo la suficiente curiosidad para usarlo en sus presentaciones, pero se asume que fue la falta de tiempo, se envió un pequeño tutorial sobre el uso de snap camera y el usarlo se dejó opcional. Google Meet también incluye características como el fondo difuminado y fondos predefinidos de patrones o imágenes como playa, cocina, biblioteca, sala, oficina etc. Con la salvedad que solo se pueden usar si se usa como navegador Google Chrome.

En cuanto a la duración se podía utilizar el tiempo completo de la clase que se distribuía en dos partes, una hora de teoría y otra hora de demostración e interacción. En el apartado de teoría se hacía comprobación de lectura por medio de preguntas generadoras que contestaban a) libremente b) por los alumnos en video c) por los alumnos en la lista de participantes, el mínimo requerido era tener micrófono, algunos no lo tenían y mandaba su respuesta al chat. Cuando a pregunta expresa a un alumno no contestaba en el chat y sin micrófono (y la mayoría de la veces con la cámara apagada), había la fuerte sospecha de que estaban en otra situación distante, como por ejemplo acompañando a un familiar en un consultorio u hospital o conectado desde su trabajo. De acuerdo al diseño instruccional de la asignatura, no solo se cubrían temas teóricos, sino que en especial la primer unidad, estaban planteados crear un portafolio con diferentes herramientas web, por lo cual, la otra hora se compartía pantalla con presentaciones para los temas teóricos y la otra hora estaba diseñado un ejercicio para plasmar su investigación del tema usando una herramienta web del tipo Canva o un mapa conceptual. O se realizaba una práctica colaborativa con Microsoft OneDrive, para Excel en línea, donde varios alumnos podías participar al mismo tiempo, asignando celdas a resolver por alumnos y resaltando en amarillo cuando estuvieran correctas y con fondo rojo cuando no lo eran. De utilidad, pero por cuestiones técnicas solo la mitad de los alumnos entraban y me llamaba la atención y que aún fuera del horario de clases los alumnos trabajaban en el archivo compartido, lo cual me confirmo que era una herramienta útil. Cuando se usaban herramientas más especializadas que no incluía la versión en línea se utilizaba compartir pantalla para que demostrarán como realizar el ejercicio. Y en especial al final de la clase, había normalmente un par de alumnos que requería asesoría personalizada, demostrando su duda compartiendo pantalla.

Otra herramienta que fue de gran utilidad para la enseñanza a distancia, fueron los videotutoriales, primero se buscaron tutoriales afines en Youtube, los cuales son buenos para los conceptos generales, pero como se tenía ejercicios específicos, había la necesidad de hacer videotutoriales específicos para esa materia ( consistente en grabar en video su pantalla 


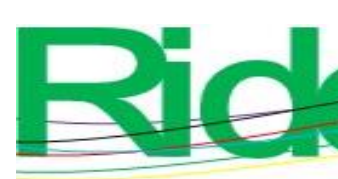

Revista Iberoamericana para la Investigación y el Desarrollo Educativo ISSN $2007-7467$

al realizar los ejercicios paso a paso de acuerdo con las instrucciones que ya venían en el curso en línea de Moodle, mismo que podía repasar varias veces una vez que estuvieran publicados en el curso en línea de moodle), junto con el diseño de cursos en línea de otras materias en el calendario 2020A me llevo a la motivación de otorgar puntos extras por elaborar material didáctico videotutoriales con la herramienta de Loom, que permite grabar la pantalla y el audio ( y/o video) que a pesar de estar en inglés, es sencilla de usar y es gratis con fines académicos, de lo contrario solo permite videos de 5 minutos. Una vez terminado el video se sube rápidamente a la nube y con un enlace se puede compartir a todos. Y con ello pude obtener los videotutoriales de procesador de palabras, yo pude realizar los de hoja de cálculo, les podía dejar que revisaran previo a la clase el videotutorial y que me lo demostraran en línea en Microsoft OneDrive (Excel en Línea) y ahorraba tiempo en tener que volver a explicar varias veces lo mismo. Se pudo reforzar los puntos débiles y debido a que la herramienta de Loom permite ver el número de reproducciones, tiene un componente social, donde los alumnos me hicieron llegar comentarios y poner reacciones de me gusta o aplausos, además me da una idea de la cantidad de alumnos que había revisado previamente el videotutorial (entre $25 \%$ y el $50 \%$ ). Los videos los podían revisar previo al ejercicio y los comentarios de los alumnos a final del semestre, indicaron que le sirvió para autogestionar mejor su tiempo, repetir las veces que fuera necesario y fuera del horario de clase las instrucciones del ejercicio. Con un alumno interesado por cada grupo se cubrieron las 4 prácticas de procesador de palabras en el ciclo 2020 A, en uno en particular se tuvo que repetir porque la alumna uso otra herramienta que mostro una marca de agua grande y un archivo pesado que tuvo que subir lentamente.

Ante el problema de que la configuración del ruteador personal del cortafuegos se cambió a alto, se bloqueó incluso la herramienta de Google Meet y entonces pasamos al plan B (en la búsqueda de herramientas de videoconferencia, tenemos el respaldo institucional de Cisco Webex, donde previamente a la falla, se había socializado en el curso en línea mi dirección para videoconferencia y la instrucción de tenerla instalada como herramienta de respaldo. Al momento de utilizarla tuvo las siguientes características:

Cisco Webex permite la programación sencilla de reuniones con un enlace personal, tiene fondos de pantallas predefinidos que se ven profesionales, permite grabar la sesión tanto en un archivo en la computadora, como en la nube. Solo que para compartir la pantalla se debe solicitar, es como si se "pidiera la palabra", tiene múltiples vistas para destacar o a los participantes o al contenido de la pantalla compartida y no tiene restricciones de tiempo. Con 

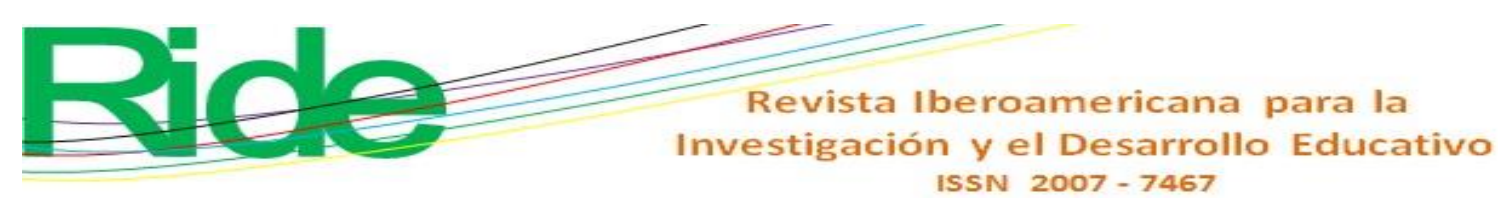

esta herramienta se participó también en un congreso de capacitación docente y ampliamente estuvimos 250 personas, pero se debe de establecer la política de micrófonos apagados, porque pueden interrumpir al Orador principal (en Google Meet se apagan automáticamente cuando hay múltiples usuarios conectados).

El plan C, otra herramienta institucional disponible Microsoft Skype, permite fácilmente establecer una foto de fondo virtual aún sin pantalla verde. Compartir pantalla, salón de charla. No lo he usado aún en clases por 2 motivos, primero por el uso principal de Google Meet para videoconferencia y la cuenta de correo institucional principal es de Google, la de Microsoft se tiene que solicitar y depende del trabajo se liberan, y no se disponía de las direcciones de correos de los 100 alumnos, que, si sucedía con las de Gmail, almacenada junto a las listas de asistencia en el sitio institucional.

\section{Resultados}

Dificultades técnicas: de acuerdo con un diagnóstico inicial, utilizando un cuestionario de Microsoft forms en línea, se pudo encontrar que el promedio de equipo de cómputo es procesador de hace 10 años, computadoras compartidas y un $20 \%$ de los alumnos se conectan vía datos. Se tomo como referencia el laboratorio presencial que tenía instalado Microsoft office 2016, pero varios alumnos tenían versiones más antiguas y lamentablemente 2 alumnos tenían chromebooks que solo pueden correr la versión en la web de Microsoft Office. Unos de los retos enfrentados en el uso de la videoconferencia fue que pocos alumnos mantenían su cámara prendida, para una mejor retroalimentación del lenguaje corporal. Entre las causas posibles de ese comportamiento esta que varios alumnos trabajaban, y tomaban las clases desde su trabajo, en trayecto en el camión (uno me comento), en espera en un consultorio médico (esperando a un familiar) y en el horario de las $7 \mathrm{am}$, varias alumnas preferían tener la cámara apagada y algunos en obscuro para no despertar a familiares si estaban compartiendo la habitación. Otro factor clave, considero fue que muchos alumnos desconocían las posibilidades del software para sustituir el fondo por una imagen fija. El ciclo 2020A el equipo de bajo rendimiento también afecto al autor con una computadora de escritorio de hace 10 años, sin cámara ni micrófono y una portátil con Mac OS de hace 5 años. Además de problemas técnicos con internet. Hizo que se grabaran videotutoriales sin voz, lo cual extrañaba a los alumnos, haciéndolo parecer incompleto. Los tutoriales en el primer ciclo se centraron en las herramientas web, más que en Microsoft office para Mac OS del cual por cierto me di cuenta de que hay contenido muy limitado en Youtube. 


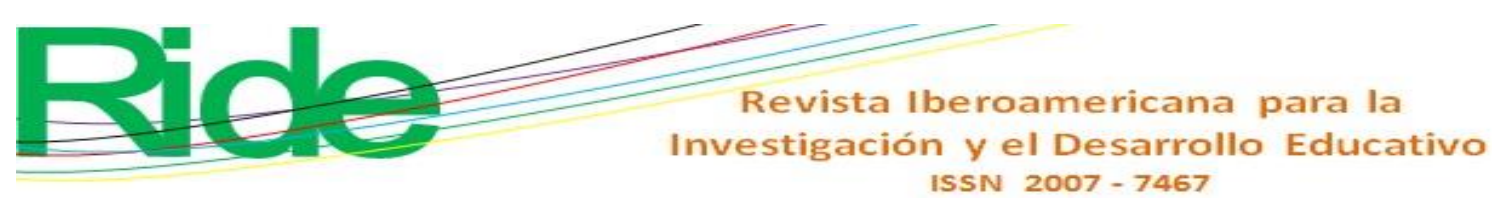

Según los comentarios con los alumnos, sus computadoras eran de hace 10 años, y en algunas ocasiones no tenían licencias legales de Microsoft office, con lo cual es uso de un laboratorio presencial sirve como un "uniforme" estandarizando el equipo entre los alumnos para un aprovechamiento óptimo.

En la unidad de presentaciones se ocupaba grabar una presentación en video, yo mismo me capacité en Prezi Video (llevaba 8 años de hacer solo presentaciones sin video), Loom (para crear videotutoriales y material didáctico por parte de los alumnos y profesores), Snap Camera (para cambiar el fondo del profesor de manera divertida) a través de tutoriales de YouTube en el calendario 2020A (al tiempo que tuve que diseñar 2 cursos en línea en Google Classroom de otras 2 materias diferentes). La mayoría lo pudo realizar.

Otra ventaja del uso de los laboratorios presenciales es la disponibilidad del internet, que en la ciudad parece común con el Wifi, pero en poblaciones menores pueden depender más de los datos. Y debido a la situación económica de muchos alumnos, que trabajan y que incluso ayudan a sus papás, se les complica pagar los datos del celular. Hubo un $20 \%$ que tuvo dificultades porque vivía en el interior del estado en un pueblo pequeño, una alumna se graba con su celular y lo subió a YouTube, otro fue a un ciber (en especial si tenían un Chromebook), otros pidieron una computadora con un familiar y hasta con un vecino.

Otra dificultad de la videoconferencia fue el disponer de tiempo en un equipo compartido, dado que todos los hermanos estaban en clases por internet y no todos tenían una computadora personal, además del lugar de estudio, un alumno refirió estudiar con primos menores de edad haciendo ruido en un patio anexo y que no lo lograban hacer concentrar.

Desde el inicio de curso se dio un medio de comunicación alternativo vía Twitter y 50\% de los alumnos lo usaron para sus dudas desde sus dispositivos móviles y $50 \%$ lo hicieron a través del curso en línea de Moodle típicamente desde sus portátiles. También se les ofreció la posibilidad de tramitar su cuenta UdeG Live para con su correo Microsoft institucional tener un office 365 académico y se promovió el uso de LibreOffice para los que no tuvieran una licencia válida y mientras llegaba su cuenta UdeG Live, además se ofreció la posibilidad de usar el office desde su celular típicamente Android.

A los alumnos que no tenían micrófono se les hacía la invitación a usar unos manos libres, alguno dijo tener problemas con la tarjeta de sonido quemada, por lo cual cuando no funcionaba su micrófono se pasaba a la participación vía el chat de la herramienta Google Meet que además era usada para indicar cuantos estaban presentes y para remarcar los avisos importantes y si había algún enlace que destacar como por ejemplo los archivos compartidos. 


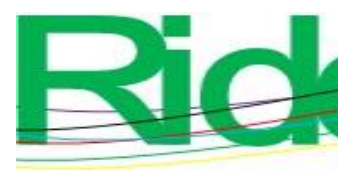

Revista Iberoamericana para la Investigación y el Desarrollo Educativo ISSN $2007-7467$

Pero si llegaban tarde no podía ver esos datos y debía revisarlos en los avisos del curso en línea.

Para el apartado de los datos se sugirió hacer cambio de portabilidad a otra compañía para tener más datos, o utilizar un proveedor de red compartida que tienen los planes más agresivos de valor/precio, además de darles a conocer los Operadores Móviles Virtuales (OMV) que normalmente tienen ofertas atractivas. En mi caso particular utilice 2 proveedores de internet móvil y en una ocasión por problemas técnicos con el Internet utilice $800 \mathrm{Mb}$ en una hora de videoconferencia con un grupo vía datos. Disponía de plan de datos de 20 a $40 \mathrm{~Gb}$ al mes y otro proveedor con $36 \mathrm{~Gb}$ de saldo acumulado y la opción de compartir datos a la portátil vía un punto de acceso inalámbrico (hotspot). Pero por lo vulnerable de la situación de los alumnos, hubo algunos que si batallaba por conseguir los datos.

\section{Discusión}

Los hallazgos reflejan que hubo un aumento súbito del uso de videoconferencias a partir de la pandemia por COVID-19,muchos profesores tuvieron que revisar varias herramientas antes de encontrar la más adecuada a su forma de trabajar, los profesores tuvieron que capacitarse en múltiples áreas relacionadas con el video, no solo en la etiqueta de las videoconferencias, su planeación, desarrollo y evaluación sino en la curación de materiales de video, el desarrollo de cursos en línea bajo la marcha en el ciclo 2020A (primavera), desarrollo de videotutoriales específicos por el profesor y motivación a la elaboración de materiales didácticos por parte de los alumnos con motivo de optimizar el tiempo. Anteriormente se utilizaba videoconferencias en entornos especializados de educación a distancia y posgrados y este año se popularizo para todas las materias y niveles desde educación básica a posgrado, en materias tecnológicas y no tecnológicas. Y muy probable vaya a ser considerada una competencia básica en lo futuro no solo para los profesores, sino para los profesionales en general y del área económico-administrativa en particular, donde me desenvuelvo. Prueba de ello la actualización en prezi video a los alumnos de primer semestre en la unidad de presentaciones de la asignatura de tecnologías de la información. Como debilidad latente, se encuentra el equipamiento de computo de profesores y alumnos, dado que esta tecnología exige más recursos de hardware, memoria y ante un panorama adverso de la economía, puede frenar el avance en su uso, pero la experiencia conseguida en este año ha sido mayormente positiva 


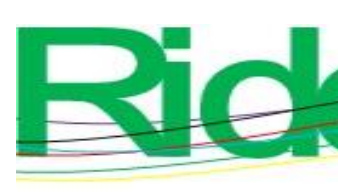

Revista Iberoamericana para la Investigación y el Desarrollo Educativo ISSN 2007-7467

\section{Conclusiones}

No es automático el paso del salón de clases a una herramienta de videoconferencia, es necesario unos prerrequisitos de entrada. Como los son el equipo, los programas y la conexión a Internet (que proporciona de entrada el laboratorio físico), la capacitación en las herramientas informáticas en especial en la videoconferencia (como por ejemplo como cambiar su fondo por una imagen) y soporte técnico previo a la videoconferencia. El lugar de trabajo libre de distracciones y con una computadora individual, no solo para las clases, sino en tiempos posteriores para realizar la tarea. Y debido a la situación económica y que muchos trabajaban o apoyaban en sus familiares, ni el tiempo de dedicación estaba asegurado, tenían que trabajar, trasladarse, apoyar a sus familiares (tal ves hasta hacer el quehacer mientras estaban en clases). A pesar de que las instrucciones eran leer los materiales previo a la clase para dialogar del tema, en ocasiones se tenia que presentar los temas y eso podía aburrir a los que si dedicaron tiempo en revisarlos. Para demostrar los procedimientos eran mejor los videotutoriales grabados, que se debían generar bajo la marcha aumentando el trabajo, la novedad de usar Onedrive para trabajo colaborativo, hacia que solo participaran la mitad de grupo por cuestiones técnicas, y los temas cargados con tiempo reducido no facilitaban el aprovechamiento del tiempo. En varias ocasiones la fecha de la clase cayo en día no hábil y en la segunda clase de trabajo en casa se fijo videoconferencia o examen y muchos no pudieron asistir por su trabajo.

Las dificultades entre otras consistieron en tener la conexión de internet, el equipo para hacer las tareas, el tiempo disponible para clases y las tareas y la autogestión para realizar las tareas de las 6 materias que llevaban en el primer semestre.

Hace 20 años que autor tomo clases en una Universidad en línea nunca se usó las videoconferencias, la tecnología ya estaba, pero ocupaba que madurará en características y en equipamiento. Conforme ha evolucionado el tiempo también los dispositivos móviles permiten videoconferencias, soluciones como Messenger, WhatsApp (que permiten 8 usuarios simultáneos) se han añadido a las ahora clásicas Microsoft Skype, Cisco Webex, Google Meet (antes Hangouts) y zoom. Si bien es una herramienta útil no es la única que se debe utilizar, aún en materias teóricas, para la educación a distancia.

En ciclo 2020 A, hizo falta tiempo, seleccionar la mejor herramienta de videoconferencia y elaboración de material didáctico en forma de videotutoriales, se apoyó en parte con los alumnos para generar videotutoriales con la herramienta Loom. Para el Fondo virtual se usó Snap Camera. 


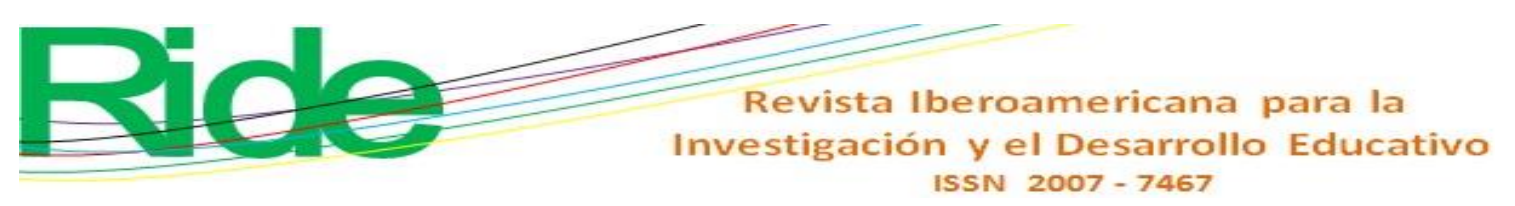

La videoconferencia es una parte de la estrategia docente junto con los cursos en línea, los videotutoriales, las herramientas de aula invertidas, los simuladores y las herramientas de colaboración.

En el caso de la educación a distancia con videoconferencia es muy importante tener la planeación del curso por adelantado y los materiales didácticos para que puedan revisarlos previo a la clase, además de que es necesario la motivación de los estudiantes y su autogestión para poder organizar sus tiempos y lugares de estudio. Para diseñar una estrategia de uso de videoconferencia se requiere una planeación previa de las sesiones, programando la fecha y en enlace y darlo a conocer a más tardar el inicio del curso, debe incluir la etiqueta de esta, por ejemplo, tener las cámaras prendidas, micrófono apagado y utilizar el chat cuando tengan problemas técnicos como falla de micrófono y el profesor para anotar puntos importantes, así como enlaces a archivos colaborativos y apuntes breves. En la planeación también es importante seleccionar la herramienta principal y la herramienta de respaldo y enviar al curso en línea o por correo un tutorial del uso de la herramienta y de preferencia el enlace para su descarga, tanto en la computadora como el celular.

Durante la sesión, es recomendable más que solo exponer una presentación (tomando en cuenta un nivel de licenciatura) es importante hacer preguntas generadoras sobre recursos que debieron haber leído previamente, así como recursos de videotutoriales y es útil pedir permiso para grabar la sesión para referencia posterior, especialmente en los exámenes. Para nombrar lista se puede desde tomar foto a la lista de asistentes hasta usar complementos de Chrome para nombrar lista en Google Meet. Es muy importante hacer participar a todos y no solo a los que tienen la cámara prendida y si alguno se tarda en responder dejar la opción para que anoten su respuesta en el chat. Para materias prácticas y de uso de herramientas ofimáticas he encontrado de utilidad el uso de Microsoft OneDrive para colaborar en tiempo real y anotar comentarios sobre el ejercicio.

Tener cuidado con las características específicas de la herramienta de videoconferencia al momento de guardar para poderlo usar como referencia futura. Es una buena práctica hacer disponible a los estudiantes las grabaciones de la clase.

Finalmente tener un plan de emergencia, que hacer en caso de: 1) tener fallas en el Internet fijo (un plan de datos, puede ayudar de respaldo) 2) tener problemas con la herramienta de videoconferencia (tener un plan B con otra herramienta de videoconferencia) . 3) sufrir una interrupción de zoombooming. (evitando publicar la dirección por redes sociales) 4) tener problemas con la luz (tener conectado un no-break y un medio de comunicación alternativo). 


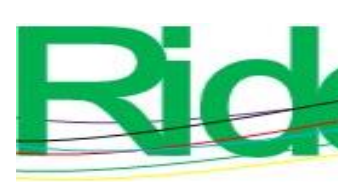

Revista Iberoamericana para la
Investigación y el Desarrollo Educativo
ISSN $2007-7467$

5) tener problemas técnicos generales (uso de software libre, aplicaciones con el celular, versión simplificada del ejercicio, etc.) Publicando una lista de preguntas frecuentes y teniendo un contacto con la mesa de soporte técnico de la universidad.

\section{Futuras Líneas de Investigación}

Como futuras líneas de investigación se propone en estudio en otros niveles educativos, medio superior, medio básico y básico. Así como el estudio del tema por medio de profesores generalistas que no impartan la materia de informática, así como evaluar las competencias de los profesores en general en el área de producción de video y videoconferencias.

\section{Referencias}

Amaya Amaya, J. (2010). Sistemas de información gerenciales: Hardware, software, redes, Internet, diseño. ECOE ediciones.

Assey, G. (2020). The Professional Business Video-Conferencing Etiquette Handbook \& Guide. Editorial Gerard Assey.

Barroso Osuna, J., \& Cabrero Almenara, J. (2014). Utilización educativa de la videoconferencia. En J. Barroso Osuna, \& J. Cabrero Almenara, Nuevos escenarios digitales (págs. 399-410). Ediciones Piramide.

Binder, j. (2012). Global Project Management: Communication, Collaboration and Management Across Borders. Editorial Gower Publishing Ltd.

Bossolasco, M. L., Martín, M. M., Ruiz Juri, M., \& Sabulsky, G. (2019). El encuentro virtual como espacio de aprendizaje: análisis de experiencias a. $8^{\circ}$ Seminario Internacional de Educación a Distancia de la Red universitaria de Educación a Distancia de Argentina (RUEDA) (pág. 12). Universidad Nacional de Jujuy.

Brown, S. (20 de junio de 2020). ¿Cuál es mejor entre Zoom y Google Hangouts? . c|net en español: $\quad$ https://www.cnet.com/es/noticias/zoom-google-hangouts-appsvideollamadas-cual-es-mejor/

Cabero Almenara, J., \& Romero Tena, R. (2010). Diseño y producción de TIC para la formación. Editorial UOC.

Calixto Molina, M. (2011). E-learning: pizarras y tabletas digitales interactivas, videoconferencias, vídeos docentes, entornos colaborativos y plataformas para la docencia online. Congreso Internacional de Innovación Docente (pág. 7). Universidad Politécnica de Cartagena. 


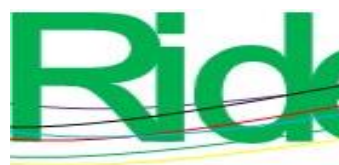

Revista Iberoamericana para la
Investigación y el Desarrollo Educativo
ISSN $2007-7467$

Caporali, E., \& Trajkovik, V. (2012). Video Conference as a Tool for Higher Education: The TEMPUS ViCES Experience. Firenze University Pres.

Castillo Holgado, M., \& Fernandez Iglesias, R. M. (2014). Diario de una pyme en Internet. Editorial Fundación COTEC.

García, É. (28 de abril de 2020). WhatsApp ya permite llamadas y videollamadas de hasta 8 personas. c|net en español: https://www.cnet.com/es/noticias/whatsappvideollamadas-ocho-personas/

García-Almiñana, D., Chester, E., \& Martinez-Sanchez, M. (2010). Promoción del empleo de las videoconferencias como elemento de potenciación de la calidad en el aprendizaje en la ETSEIAT. CUIEET 2010 (pág. 8). Editorial UPcommons.

Heller, R. M. (2010). The contribution of telepresence to support distance management. Editorial diplom.de.

Hernández Adell, I. (2016). El mundo Digital en la enseñanza: experiencias con los cursos virtuales y las aplicaciones móviles en el aula. En M. Bringas, E. Catalán, C. Trueba, \& L. E. Remuzgo, Nuevas perspectivas en la investigación docente de la historia económica (págs. 495-512). Editorial Universidad Cantabria.

Huergo-Tobar, P. L. (2015) "Estrategias de enseñanza-aprendizaje a través de videoconferencias". Rastros Rostros 17.31: 65-76. Impreso. doi: http://dx.doi.org/10.16925/ra.v17i31.1096.

Krakower, B., \& Blumengarten, J. (2020). Connecting Your Students with the Virtual World: Tools and Projects to Make Collaboration Come Alive. Editorial Routledge.

Ling, C., Balc1, U., Blackburn, J., \& Stringhini, G. (8 de septiembre de 2020). Cornell University https://arxiv.org/. A First Look at Zoombombing: https://arxiv.org/pdf/2009.03822.pdf

Lopez Curiel, R. (2014). Las TIC en el aula de Tecnología. Guía para su aplicación a la metodología de proyectos. Editorial ADP Asociación para el desarrollo del profesorado.

Microsoft presenta la versión educativa de Teams. (28 de junio de 2017). MVP Cluster: https://mvpcluster.com/noticia/microsoft-teams-educacion/

Salas-González, S. (2020). Percepciones de las personas docentes para el mejoramiento de la mediación pedagógica en el uso de videoconferencias en la Universidad Estatal a Distancia (UNED). Innovaciones Educativas, 17. 

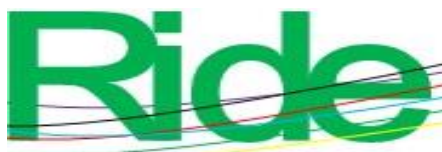

Revista Iberoamericana para la
Investigación y el Desarrollo Educativo ISSN $2007-7467$

\section{Salvador Barrera Rodríguez}

Maestro en Administración por la Universidad de Guadalajara. Master en Dirección de Empresas Digitales y Desarrollo de Negocios en Internet por la Universitat Oberta de Catalunya y Licenciado en Administración de Empresas por la Universidad de Guadalajara. Este certificado en Microsoft Excel, IBM DB2 y Computo en la nube. Es técnico programador analista. Profesor de tiempo completo con Perfil Deseable del Programa de Mejoramiento del Profesorado (PROMEP), trabaja en la Universidad de Guadalajara en el Centro Universitario de Ciencias Económico Administrativas (CUCEA). 